\title{
Engineering the oleaginous yeast Yarrowia lipolytica to produce the aroma compound $\beta$-ionone
}

Jeffrey J. Czajka', Justin A. Nathenson'1, Veronica T. Benites², Edward E. K. Baidoo², Qianshun Cheng 3,4, Yechun Wang ${ }^{5^{*}}$ and Yinjie J. Tang ${ }^{1 *}$ (D)

\begin{abstract}
Background: $\beta$-lonone is a fragrant terpenoid that generates a pleasant floral scent and is used in diverse applications as a cosmetic and flavoring ingredient. A growing consumer desire for natural products has increased the market demand for natural $\beta$-ionone. To date, chemical extraction from plants remains the main approach for commercial natural $\beta$-ionone production. Unfortunately, changing climate and geopolitical issues can cause instability in the $\beta$-ionone supply chain. Microbial fermentation using generally recognized as safe (GRAS) yeast offers an alternative method for producing natural $\beta$-ionone. Yarrowia lipolytica is an attractive host due to its oleaginous nature, established genetic tools, and large intercellular pool size of acetyl-CoA (the terpenoid backbone precursor).
\end{abstract}

Results: A push-pull strategy via genome engineering was applied to a Y. lipolytica PO1f derived strain. Heterologous and native genes in the mevalonate pathway were overexpressed to push production to the terpenoid backbone geranylgeranyl pyrophosphate, while the carB and biofunction carRP genes from Mucor circinelloides were introduced to pull flux towards $\beta$-carotene (i.e., ionone precursor). Medium tests combined with machine learning based data analysis and ${ }^{13} \mathrm{C}$ metabolite labeling investigated influential nutrients for the $\beta$-carotene strain that achieved $>2.5 \mathrm{~g} / \mathrm{L}$ $\beta$-carotene in a rich medium. Further introduction of the carotenoid cleavage dioxygenase 1 (CCD1) from Osmanthus fragrans resulted in the $\beta$-ionone production. Utilization of in situ dodecane trapping avoided ionone loss from vaporization (with recovery efficiencies of $\sim 76 \%$ ) during fermentation operations, which resulted in titers of $68 \mathrm{mg} / \mathrm{L}$ $\beta$-ionone in shaking flasks and $380 \mathrm{mg} / \mathrm{L}$ in a $2 \mathrm{~L}$ fermenter. Both $\beta$-carotene medium tests and $\beta$-ionone fermentation outcomes indicated the last enzymatic step CCD1 (rather than acetyl-CoA supply) as the key bottleneck.

Conclusions: We engineered a GRAS Y. lipolytica platform for sustainable and economical production of the natural aroma $\beta$-ionone. Although $\beta$-carotene could be produced at high titers by Y. lipolytica, the synthesis of $\beta$-ionone was relatively poor, possibly due to low CCD1 activity and non-specific CCD1 cleavage of $\beta$-carotene. In addition, both $\beta$-carotene and $\beta$-ionone strains showed decreased performances after successive sub-cultures. For industrial application, $\beta$-ionone fermentation efforts should focus on both CCD enzyme engineering and strain stability improvement.

Keywords: ${ }^{13} \mathrm{C}$ labeling, Terpenoid, Acetyl-CoA, $\beta$-carotene, Machine learning, Fed-batch fermentation, Strain stability

\footnotetext{
*Correspondence: ywang@arch-innotek.com; yinjie.tang@wustl.edu

1 Department of Energy, Environmental and Chemical Engineering, Washington University, St. Louis, MO 63130, USA

${ }^{5}$ Arch Innotek, LLC, 4320 Forest Park Ave, St. Louis, MO 63108, USA

Full list of author information is available at the end of the article
}

(c) The Author(s) 2018. This article is distributed under the terms of the Creative Commons Attribution 4.0 International License (http://creativecommons.org/licenses/by/4.0/), which permits unrestricted use, distribution, and reproduction in any medium, provided you give appropriate credit to the original author(s) and the source, provide a link to the Creative Commons license, and indicate if changes were made. The Creative Commons Public Domain Dedication waiver (http://creativecommons.org/ publicdomain/zero/1.0/) applies to the data made available in this article, unless otherwise stated. 


\section{Background}

Terpenoids are a class of secondary metabolites that have a wide-range of biochemical functions in nature, including pigments, anti-oxidants, signaling molecules, and plant defense mechanisms [1-3]. The diverse properties of terpenoids have led to their wide-spread use in the cosmetic, pharmaceutical, and fragrant and flavor industries [4-6]. $\beta$-Ionone, an apo-carotenoid (derived from a C40 terpenoid, also known as a carotenoid), is one such molecule. The chemical has an intense, woody smell and a low odor threshold [7] that has led to its utilization in industrial fragrance products, such as perfumes, personal care products, and as a flavoring agent in non-alcoholic beverages and gelatins [8]. The yearly global ionone market averaged approximately $\$ 166$ million from 2011 to 2015, but demand is expected to increase due to new emerging markets in Brazil, China, and India. In addition to the growing demand in existing market segments, $\beta$-ionone has the potential for applications in the health care and pharmaceutical markets. For example, it demonstrated the ability to inhibit breast cancer cells in vivo [9] and to limit tumor incidence in a rat cancer model [10]. Commercial $\beta$-ionone can be chemically synthesized, but such a product is less valuable than plant derived $\beta$-ionone due to recent increases in consumer desire for natural food products (i.e., natural aroma compounds have a range of 10-100 fold price increase over the chemically synthetic alternative) [11]. Natural aroma molecules can be obtained via extraction from plants [12], but low biochemical abundance makes product isolation labor intensive and costly. Raspberries, one of the most abundant sources of ionone, produce only $1.72 \mathrm{mg}$ of $\beta$-ionone $/ \mathrm{kg}$ of wet weight [7]. Increasing ionone production through growing or obtaining more crop biomass is not a feasible long-term solution due to land scarcity, crop utilization priorities, and unpredictable weather and climate change (which also introduces instability into the current supply chain) [13]. A second method for obtaining natural $\beta$-ionone involves using in vitro enzymatic processes (i.e., the biotransformation of $\beta$-carotene to $\beta$-ionone). However, the enzymatic bio-transformations often lead to a variety of undesirable byproducts $[11,14]$. These specific limitations motivate research into new reliable production processes for obtaining natural $\beta$-ionone.

Metabolic engineering is a promising alternative approach, as the terpenoid biochemical pathways and genes have been elucidated (Fig. 1) [15]. While much effort has focused on developing microbial platforms for production of terpenoids, few papers have reported aroma production. Recently, Escherichia coli was successfully engineered to produce $0.5 \mathrm{~g} / \mathrm{L}$ of $\beta$-ionone [16]. However, the US Food and Drug Administration (FDA) does not categorize $E$. coli as a GRAS microorganism.
Efforts in the GRAS yeast Saccharomyces cerevisiae have resulted in yields too low for viable industrial production (1 mg/g dry cell weight) $[17,18]$. One promising microorganism is the non-conventional yeast Yarrowia lipolytica. This GRAS species offers the potential to become a new production platform for $\beta$-ionone due to its innate metabolic capabilities [19]. Particularly, Y. lipolytica has a rich acetyl-CoA pool due to its cytosolic ATP citrate lyase (ACL) [20, 21]. Genetic tools have been established in $Y$. lipolytica [22], and several groups have successfully engineered the species to produce diverse carotenoids [2325]. Another industrial advantage of utilizing $Y$. lipolytica is its capacity to grow on a large range of carbon sources (including but not limited to: glucose, glycerol, alcohols, and fatty acids), allowing for the use of diverse and cheap feedstock [26]. In addition, the yeast's biomass can be incorporated into animal feed, providing a secondary revenue source [27]. Currently, the species is widely used in industry as a single cell protein factory and for production of products such as citric acid and peach flavor [28]. Here, we report engineering $Y$. lipolytica to produce $\beta$-ionone via overexpression (Push) of the mevalonate pathway, the introduction of three heterologous enzymes (Pull), and fermentation optimization.

\section{Results \\ Genetic engineering}

For carotenoid synthesis, the co-expression of $\operatorname{car} B$ and carRP genes from Mucor circinelloides led to production of $\beta$-carotene, confirmed by HPLC analysis. The further integration of a carotenoid cleavage dioxygenase 1 of Osmanthus fragrans (OfCCD1) into the genome led to detectable production of $\beta$-ionone (see Fig. 1 for chromatogram). $Y$. lipolytica contains a native mevalonate (MVA) pathway that operates with relatively low flux [29]. To circumvent the native regulatory network and push flux through the pathway, two heterologous genes (NphT7 from Streptomyces sp. [30] and IPI from Haemotoccus pluvialis [31, 32]) were integrated into the genome. Native genes in the MVA pathway were also overexpressed by increasing their copies in the genome (Fig. 1), as described in previous papers [29, 33-35]. Each gene was expressed under the same constitutive promoter (TEF) and terminator (XPR2).

\section{Medium optimization for $\beta$-carotene production}

$\beta$-Ionone (a volatile compound) can be difficult to capture and quantify, while $\beta$-carotene is detectable via UV Spectrophotometry [36]. Therefore, we utilized the $\beta$-carotene strain as proxy for $\beta$-ionone production as a high throughput screening of strain response to medium conditions. Yeast-peptone-dextrose (YPD) medium is used to promote $Y$. lipolytica biosynthesis, but it contains 


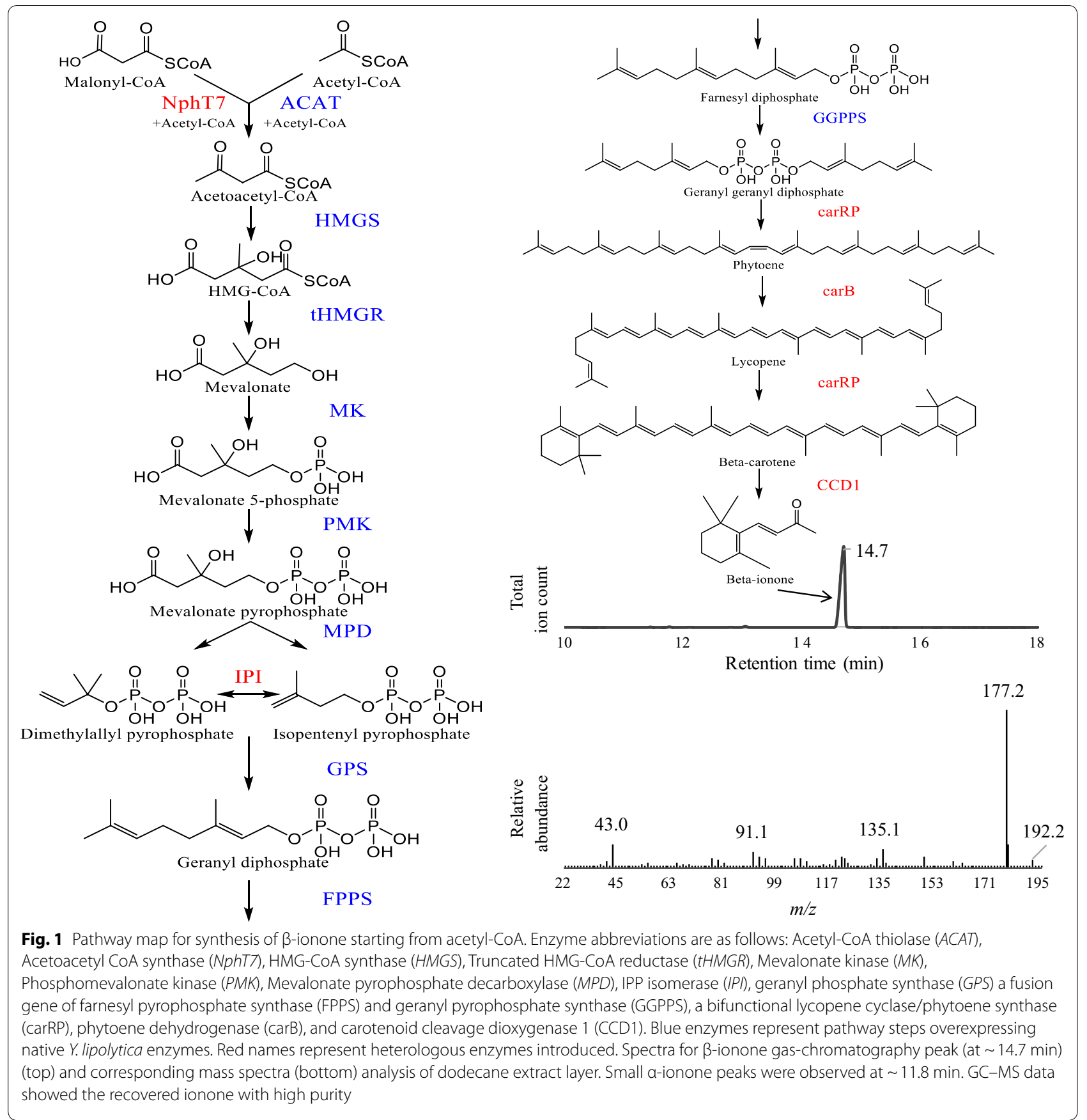

a complex mix of nutrients. To identify key nutrients that specifically contribute to $\beta$-carotene production, shaking tube medium tests in the defined Yeast-Nitrogen Base (YNB) medium supplemented with varying amino acids and carbon co-substrates were performed. Specifically, as acetyl-CoA is thought of being the limiting precursor for the MVA pathway, carbon sources that directly contribute to the acetyl-CoA pool (acetate, citrate, ethanol, etc.) were tested. In addition to revealing beneficial medium components, the outcomes could also reveal whether acetyl-CoA supply was rate limiting during Yarrowia bio-production. However, the experimental variation in medium components and biological noise made it difficult to determine which factors were beneficial (Fig. 2a, Additional file 1). Machine learning, a powerful tool for analyzing complex data $[37,38]$, was utilized to decipher the data. The trained model reached $89 \%$ prediction accuracy on the test data, while a permutation feature 

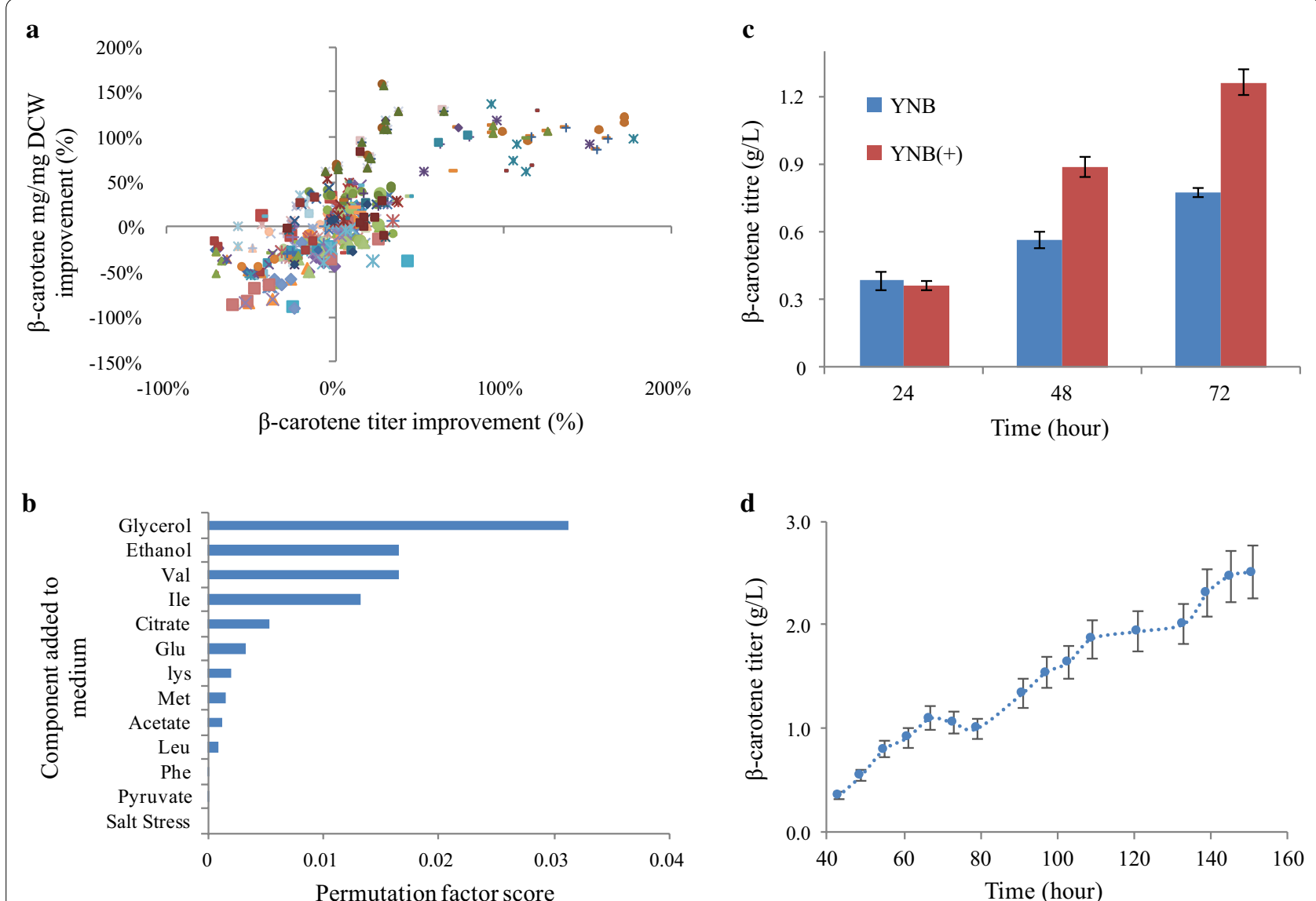

Fig. 2 Data and analysis from medium optimization experiments. a Spread of data representing the $\beta$-carotene titer and yield/DCW percent improvement relative to strains grown in the base (YNB only) medium. Each shape and color represents a separate medium condition (labels not shown due to visual limitations, see Additional file 1); $\mathbf{b}$ The feature ranking of the medium components on the predictive power of the gradient boosted tree machine learning model; c Comparison of the $\beta$-carotene titers achieved for the YNB medium and YNB(+) medium in $25 \mathrm{~mL}$ shake flask (YNB medium was supplemented with an additional $2 \mathrm{~g} / \mathrm{L}$ glucose to match the primary carbon source concentrations); $\mathbf{d} \beta$-carotene titers achieved in bench-top fermenter using a YPD medium (error bars represent measurement variations)

importance score verified the indicators of high production (Fig. 2b). Based on the model analysis, glycerol was the most beneficial component, leading to increased titers of $\sim 30 \%$. The addition of excess isoleucine and valine (whose syntheses consume acetyl-CoA) were the next most important factors. Lastly, supplementation of ethanol during the late growth phase was also beneficial.

\section{$\beta$-Carotene production in optimized medium}

With information from the high throughput shaking tubes, a $\mathrm{YNB}(+)$ medium was developed that augmented YNB medium with glycerol, valine, and isoleucine. Ethanol was also added during the mid-growth phase (see "Methods"). The $\mathrm{YNB}(+)$ medium moderately benefited $\beta$-carotene production. In shaking flask cultures, an average improvement of $43 \% \pm 7 \%$ ( $\mathrm{p}$-value $<0.01$ ) in $\beta$-carotene titer $(1.26 \pm 0.05 \mathrm{~g} / \mathrm{L})$ was achieved compared to the YNB cultures (Fig. 2c). The cells grown in defined $\mathrm{YNB}(+)$ medium showed productivity close to cells grown in YPD medium (rich in yeast and peptone). In a representative fed batch fermentation with a $2 \mathrm{~L}$ bioreactor, the cells cultured in YPD medium with glucose supplements achieved a titer close to $\sim 2.5 \mathrm{~g} / \mathrm{L}$ at $160 \mathrm{~h}$ (Fig. 2d), similar to previously reported efforts [23].

\section{${ }^{13} \mathrm{C}$-Isotopic labeling of central metabolites}

Isotopic tracing experiments were performed to gain molecular insight into the contribution of nutrient supplements to the metabolism of $Y$. lipolytica. Cell cultures were grown in the YNB medium with $\mathrm{U}_{-}{ }^{13} \mathrm{C}$ (fully labeled) glucose as the carbon source. Unlabeled secondary carbon sources or amino acids (glycerol, ethanol, isoleucine, or valine) were pulsed into the YNB cultures during the early exponential phase. The cell metabolism was quickly quenched using liquid nitrogen in the 
late growth phase, and the metabolites were extracted and analyzed via LC/MS (see "Methods"). The dilution of labeling relative to the fully labeled glucose control experiment was used to assess each component's contribution (Fig. 3). Overall, glycerol had the largest carbon contribution to the central metabolism, having 30\% or more dilution change in most metabolites (including energy molecules ATP and NADH) relative to the control. Ethanol had only minor contributions to the central metabolites; however, it contributed to the mevalonate pool since its degradation promotes cytosolic acetyl-CoA pool (not mitochondrial acetyl-CoA pool). The amino acid supplements led to minor changes in central metabolite labeling $(<10 \%)$, indicating sufficient amino acid synthesis capability in Yarrowia.

\section{In situ ionone trapping}

As previously mentioned, $\beta$-ionone is a volatile compound with limited aqueous solubility $(169 \mathrm{mg} / \mathrm{L}$ in water at $25{ }^{\circ} \mathrm{C}$ [39]). In a control experiment, $80 \%$ of $\beta$-ionone (pulsed at relevant production titers of 0.5 and $1 \mathrm{~g} / \mathrm{L}$ ) was lost from the aqueous medium in our fermenter setup within 1 day. Since $\beta$-ionone has been reported to be soluble in alcohol and organic solvents [40], an organic compound was explored as means to capture the product. Dodecane is a relatively non-volatile compound that has previously been used as an organic overlay to trap volatile terpenoids during cell fermentations, including $\beta$-ionone $[18,41,42]$. Thus, we investigated the ability of dodecane to partition and trap $\beta$-ionone during our bioreactor growths. $0.5 \mathrm{~g} / \mathrm{L}$ of $\beta$-ionone was pulsed at three separate times into the $2 \mathrm{~L}$ fermenter containing a $10 \%$ by volume dodecane layer and YPD medium to obtain biologically relevant concentrations in the range of $0-1.5 \mathrm{~g} / \mathrm{L}$ (Fig. 4a). Our analysis determined that dodecane phase captured $74 \%$ of $\beta$-ionone, while $2 \%$ of compound remained in the aqueous phase, leading to an overall capture efficiency of $76 \%$ (Fig. $4 \mathrm{~b}$ ). It was found that the dodecane addition did not adversely affect the $\beta$-ionone strain's growth in shaking flask (Fig. 4c) and that the time of addition ( 0 vs $24 \mathrm{~h}$ of growth) did not lead to a significant increase ( $\mathrm{p}$ value $=0.16$ ) in the titer achieved (Fig. 4d). Therefore, all ionone fermentations were performed with the dodecane present in the medium from the start.

\section{$\beta$-Ionone production in fed-batch fermentations}

Fermentation of $\beta$-ionone strains occurred in a $2 \mathrm{~L}$ vessel operating in fed-batch mode for $140 \mathrm{~h}$. The dodecane overlay was kept at $10 \%$ of the working volume. $\beta$-Ionone was measured in three components; the dodecane overlay, the medium, and the intracellular component. Attempts to separate any volatized $\beta$-ionone by bubbling the off-gas through one or two

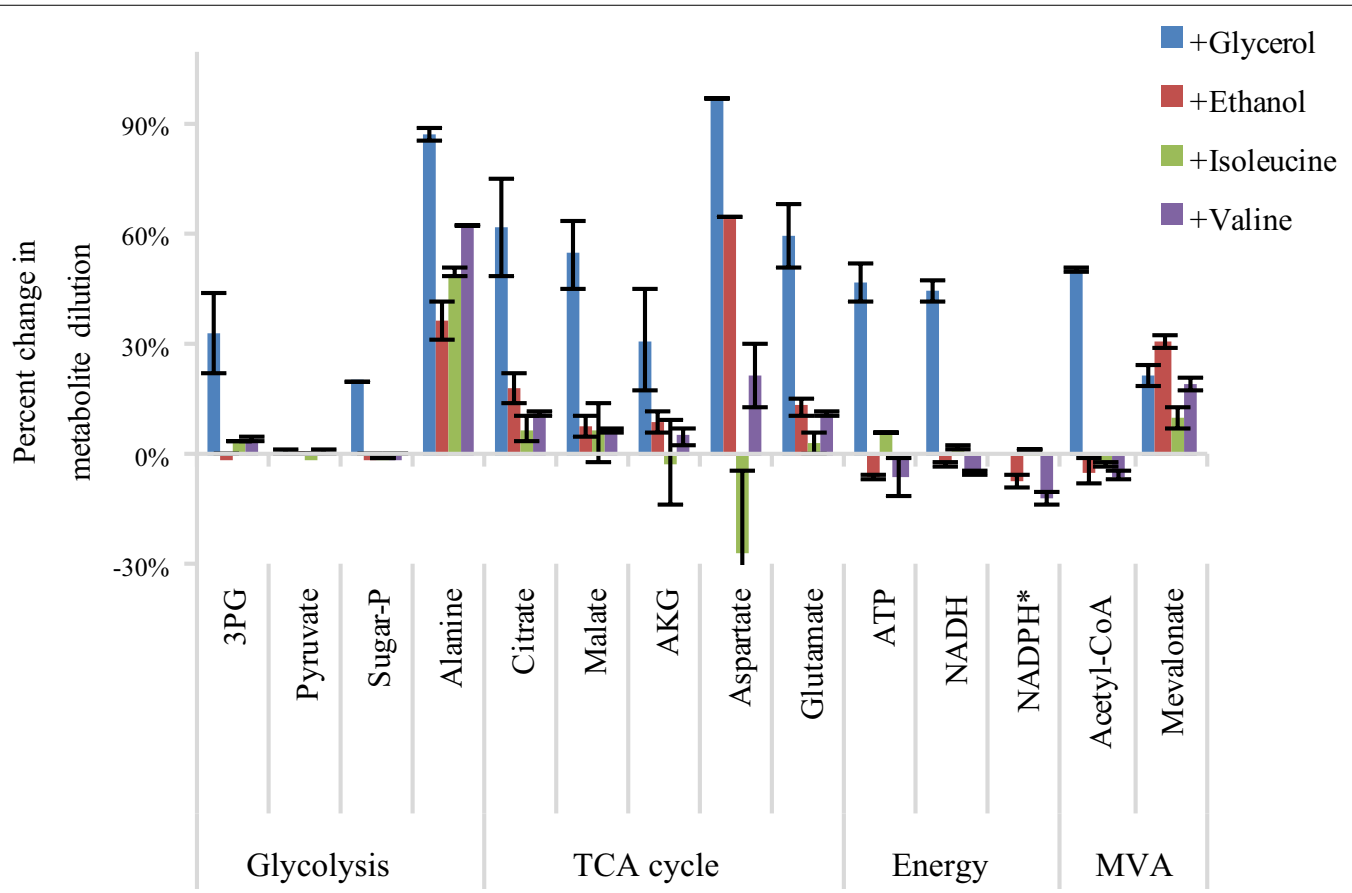

Fig. 3 Isotopic labeling results. Bars show the percent change in the metabolite dilution relative to the control (base line medium supplemented with $U-{ }^{13} \mathrm{C}$ glucose), which is calculated by: Metabolite labeling dilution percentage $=\left(M_{\text {control }}-M_{\text {test }}\right) / M_{\text {control }} \cdot M_{\text {control }}$ is Metabolite ${ }^{13} C$ percentage from the fully labeled glucose culture; $M_{\text {test }}$ is metabolite ${ }^{13} \mathrm{C}$ percentage from tested conditions. Error bars represent the standard deviation $(n=2)$ 

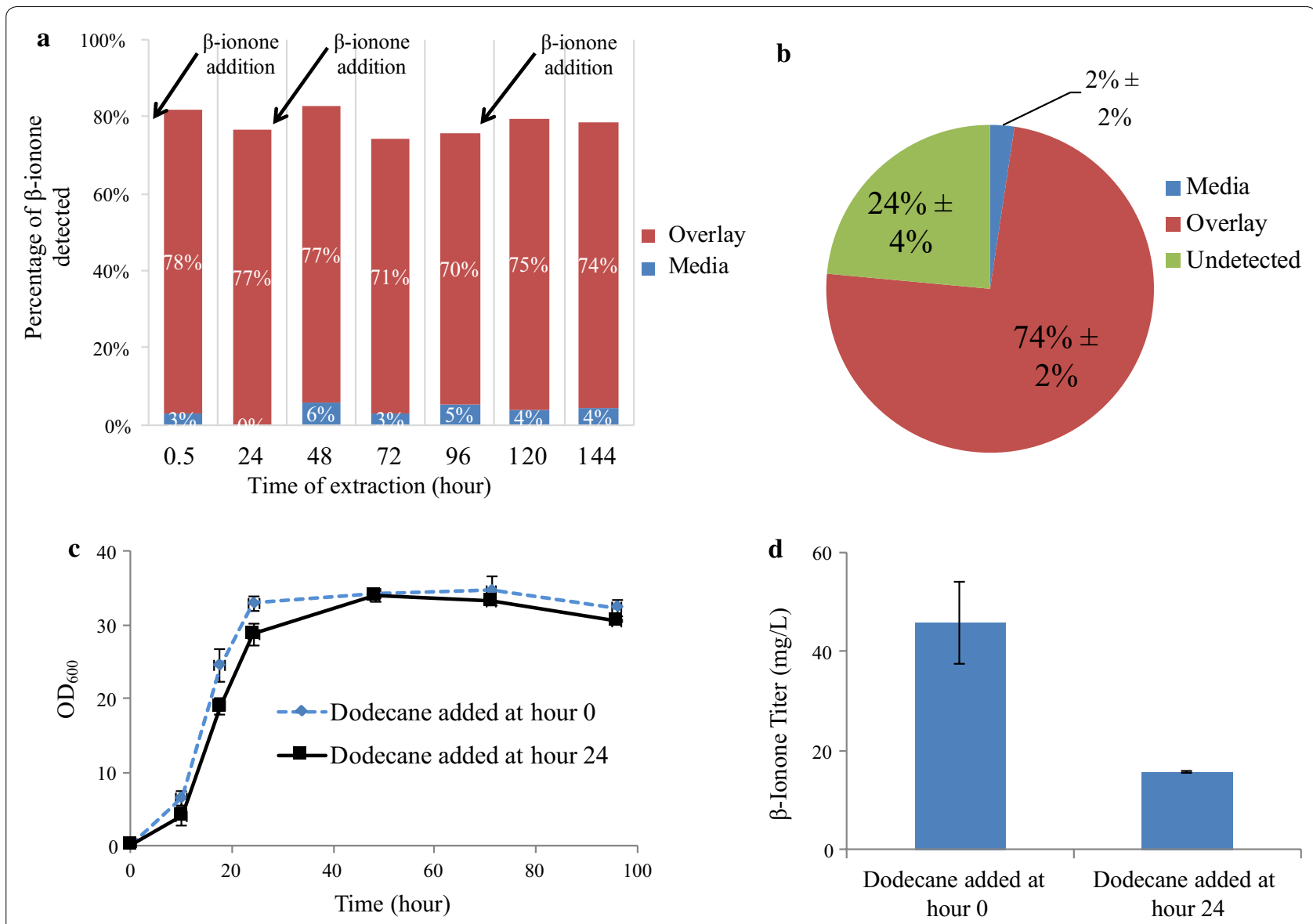

Fig. 4 Extraction efficiencies and shaking flask fermentation. a Graph showing the relative stability of the extraction method over time; $\mathbf{b}$ The percentage of $\beta$-ionone retained $24 \mathrm{~h}$ after a pulse in the $2 \mathrm{~L}$ fermenter, with an air flow rate of $0.5 \mathrm{SLPM}$ at a pH 5.5; c Growth curves of the $\beta$-ionone strain in shaking with a 10\% dodecane overlay. The dodecane was either added at immediately ( $0 \mathrm{~h}$, dashed blue line) or after $24 \mathrm{~h}$ (sold black line); d Measured $\beta$-ionone titers in shaking flask from $\mathbf{c}$ after $24 \mathrm{~h}$. Error bars represent standard deviation ( $\mathrm{n}=3$ in $\mathbf{a}, 2$ in $\mathbf{c}$ and $\mathbf{d}$ )

stages of chilled dodecane led to no appreciable absorption ( $<1 \mathrm{mg}$ captured). At the end of the fermentation, a highest titer of $380 \mathrm{mg} / \mathrm{L}$ was achieved in the glucose fed fermentation (Fig. 5). The co-fed fermentation of additional glycerol or optimized medium components (glycerol, ethanol, isoleucine, and valine) did not obtain higher $\beta$-ionone titers vs the glucose fed fermentation. Interestingly, despite the volatility of the compound, a significant portion of the total $\beta$-ionone titer was retained intracellularly at $140 \mathrm{~h}(\sim 30 \%$, Fig. $5 \mathrm{~d})$. This intracellular portion increased as $\beta$-ionone was produced throughout the fermentation. Y. lipolytica is known to utilize complex lipid bodies to store lipids and fat soluble compounds, in which the intracellular ionone may have partitioned [43]. The $\beta$-ionone strain also showed little accumulation of $\beta$-carotene $(1.8 \pm 0.3 \mathrm{mg} / \mathrm{L}$ at $140 \mathrm{~h})$ during the fermentations. Overall, the achieved ionone titer was about tenfolds lower compared to the $\beta$-carotene strain's titers. Rather than low MVA pathway flux or carotene synthesis, the last enzyme OfCCD1 is possibly the key bottleneck due to poor activity or non-specific cleavage of $\beta$-carotene.

\section{Engineered strain stability}

For industrial bio-manufacturing using engineered hosts, strain stability is essential for commercial viability. Here, we tested the production stability of the engineered $\beta$-carotene and $\beta$-ionone strains by continuously sub-culturing them on YPD plates (twice) and in shake flask cultures ( 100 doubling times). As shown in Fig. 6, both strains became unstable after extended sub-culturing. The $\beta$-ionone strain maintained its production level over more generations than the $\beta$-carotene strain, possibly due to its lower productivity and product accumulation (and thus lower metabolic burden and stress). 

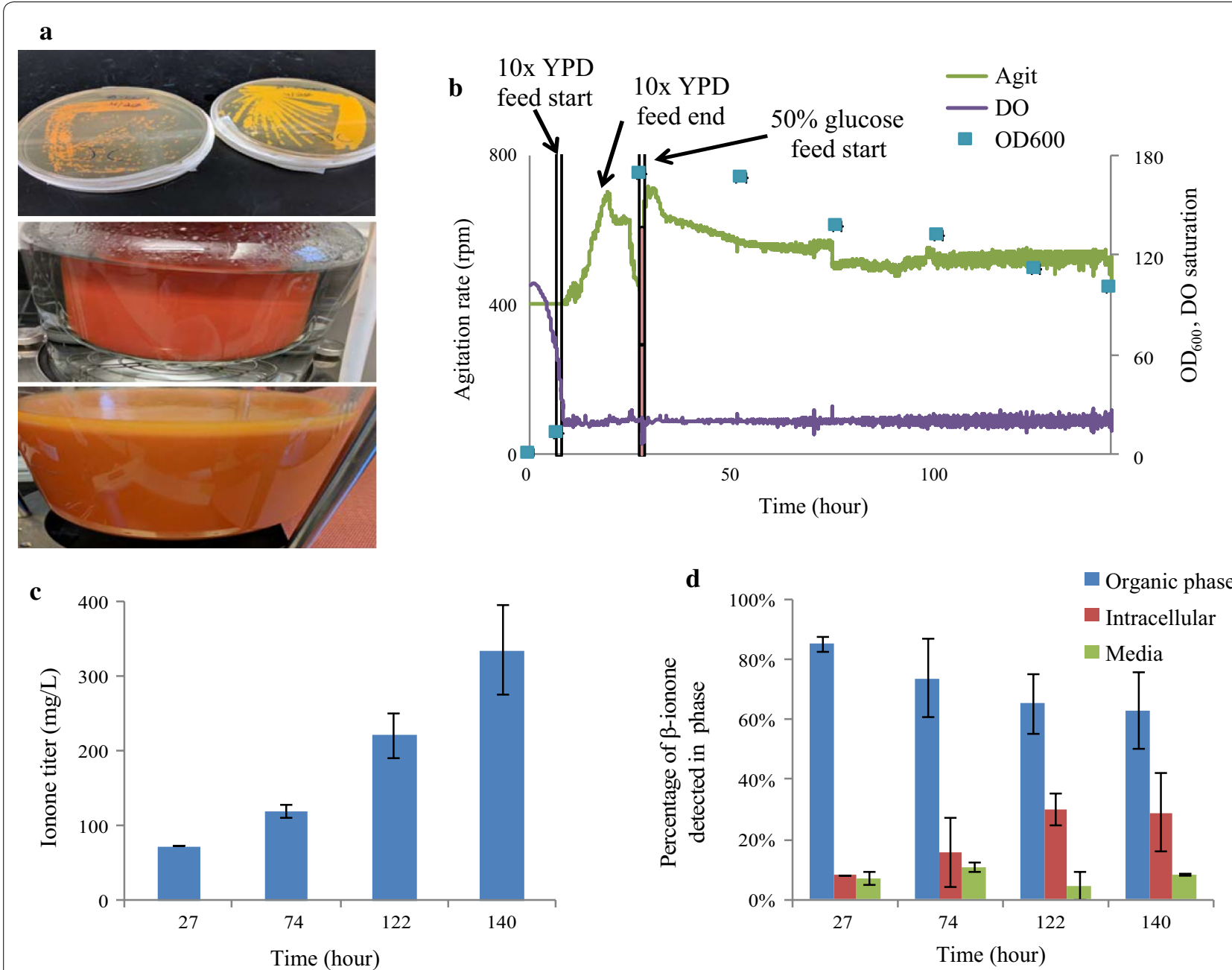

Fig. 5 Strain fermentations a (Top) Comparison of $\beta$-carotene strain (left) and $\beta$-ionone strain (right) grown on YPD plates, (middle) pigmentation of bioreactor at the end of $\beta$-carotene strain fermentation (dark orange color), and (bottom) $\beta$-ionone strain fermentation (light orange color); $\mathbf{b}$ Representative fermentation parameters; $\mathbf{c} \beta$-ionone fermentation profile; $\mathbf{d}$ Distribution of $\beta$-ionone extract from each phase analyzed (intracellular, dodecane overlay, and aqueous medium)

\section{Discussion}

Metabolic engineering often employs Push (increase precursor supply) and Pull (improve end-product enzyme functions) strategies to enhance productivity. Acetyl-CoA is a key terpenoid precursor needed to push flux through the over-expressed MVA pathway. While $Y$. lipolytica has large quantities of cytosolic acetyl-CoA due to its ACL $[20,21]$, many important competing pathways, including fatty acids and lipids synthesis, can quickly drain the available resources. Interestingly, the supplementation of citrate and acetate (direct carbon contributors for cytosolic acetyl-CoA) did not show significant improvement in the $\beta$-carotene producing strain's titer (Fig. 2a). Ethanol supplementation did enhance the titer, but isotopic tracing via LC-MS (Fig. 3) only indicated a small contribution to acetyl-CoA pools. Isotopic data indicates that the $\beta$-carotene strain had a sufficient cytosolic acetyl-CoA pool for both biomass and product production, allowing for relatively high titers of $\beta$-carotene.

Thus, evidence points to the last enzymatic step in $\beta$-ionone production, catalyzed by the CCD1 enzyme, as the rate-limiting step (i.e., glycerol supplementation increased $\beta$-carotene strain's titers but not the $\beta$-ionone strain's titers). Similar issues were previously reported with $\beta$-ionone engineering efforts in $E$. coli, where the authors discovered the OfCCD1 enzyme had low expression and non-specific cleavages of the precursors phytoene and lycopene [16], which is consistent with poor conversion of $\beta$-carotene to ionone in our engineered strain. Increasing the solubility and expression of OfCCD1 through a fusion-protein partner did not lead 


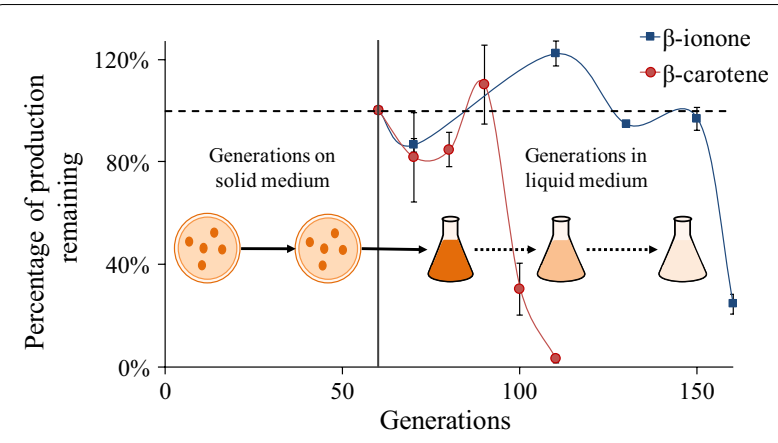

Fig. 6 Depiction of strain stability subculture experiments and results versus the number of generations accumulated. There was no selection pressure in the agar plate medium, and we estimated 30 doubling times (i.e., generations) for engineered strain to form colonies from each plating based on the mass of a single cell [54]. The engineered strain was plated twice before liquid subcultures. Solid vertical line indicates transition from solid plate growths to liquid medium

to a significant increase of $\beta$-ionone production by $E$. coli [16]. Furthermore, only slight improvement of production was achieved through introduction of different CCD1 enzymes from either Vitis vincefera (VvCCD1) or Petunia hybrid (PhCCD1) in E. coli [16]. This paper further supports the CCD1 enzyme as a key target for future engineering efforts. Interestingly, the $E$. coli system containing the OfCCD1 enzyme reached a $\beta$-ionone titer of $32 \mathrm{mg} / \mathrm{L}$ in shaking flask conditions [16]. Our Y. lipolytica shaking flask titers nearly doubled the previously obtained titer $(60 \pm 8 \mathrm{mg} / \mathrm{L})$. However, our larger scale $2 \mathrm{~L}$ fermentation did not surpass the $E$. coli titer in a $250 \mathrm{~mL}$ mini-bioreactor $(380 \mathrm{mg} / \mathrm{L}$ vs $500 \mathrm{mg} / \mathrm{L})$, possibly due to higher loss of ionone from bioreactor off gas as well as the instability of carotenoid yeast strain during process scale-up.

Yeast engineering for aroma production is still challenging with many potential bottlenecks. Inefficient enzyme steps often limit productivity while the introduction of long heterologous enzymatic pathways cause metabolic burdens, even with genome integration [44]. $\beta$-Ionone production requires further engineering and optimization efforts to improve the rate and specificity of the $\mathrm{CCD} 1$ catalyzed enzymatic transformation of $\beta$-carotene to $\beta$-ionone. In addition, the high levels of carotenoid intermediates may introduce cell stress, leading to strain instability after successive subcultures. One approached aimed at increasing production and minimizing metabolic burden is modular engineering, through which small sub-sections of the pathway are individually optimized [45-47]. This approach can potentially improve $\beta$-ionone strain stability for the industrial applications. Finally, the $\beta$-ionone extraction efficiency using dodecane may be over-estimated during bioreactor operations (i.e., due to complex interactions among gas/liquid/solid interfaces).
Therefore, improving the efficiency of the product extraction and capture is necessary to ensure successful production on an industrial scale. We will continue work on enzyme engineering to address the encountered issues as well as improving the means of product extraction and capture, aiming to achieve the break-even point of $1 \mathrm{~g} / \mathrm{L}$ for commercial production [14].

\section{Conclusion}

Yarrowia lipolytica is a promising industrial microbe that has the potential to be utilized for production of terpenoid compounds. Here, to our knowledge, we have successfully engineered $\beta$-ionone production in Y. lipolytica for the first time. We also identified the last enzymatic step in $\beta$-ionone production as a key bottleneck as further engineering target.

\section{Methods}

\section{Chemicals, medium compositions, and cell growths}

Yeast nitrogen base (YNB) and synthetic drop-out complete mix (SD drop-out) were purchased from US Biological Life Sciences (Salem, MA, USA). ${ }^{13}$ C-labeled substrates were purchased from Cambridge Isotope Laboratories (Tewkbury, MA, USA). Glucose enzymatic kits were purchased from Boehringer Mannheim. All other chemicals and enzymatic kits were purchased from Sigma-Aldrich (St. Louis, MO, USA). The Y. lipolytica strain CLIB138 (MatB, leu2-35, lys5-12, ura3-18, xpr2LYS5) was purchased from CIRM-Levures (Thiverval-grignon, France) and used as host cells in the study. $Y$. lipolytica was grown in YPD medium (per liter: $10 \mathrm{~g}$ yeast extract, $20 \mathrm{~g}$ peptone, $20 \mathrm{~g}$ of glucose) or YNB medium (per liter: $6.7 \mathrm{~g}$ yeast nitrogen base with ammonia sulfate, $1.72 \mathrm{~g}$ SD drop-out supplemented with 10 or $20 \mathrm{~g}$ of glucose). Strains were maintained on YPD agar plates (YPD medium supplemented with $20 \mathrm{~g} / \mathrm{L}$ of agar). All growth conditions followed a similar procedure. A single colony (from fresh plates) was used to inoculate seed cultures in the YPD medium (containing $10 \mathrm{~g} / \mathrm{L}$ glucose) and grown overnight at $\sim 30^{\circ} \mathrm{C}$ in shaking flasks (250 rpm). The seed culture was used for inoculation of sub-cultures (initial $\mathrm{OD}_{600}$ of $\left.\sim 0.03\right)$. For $\beta$-ionone strain cultures, a dodecane layer ( $10 \%$ by volume) was employed for product trapping. Growth was monitored by measuring $\mathrm{OD}_{600}$ using a UV-vis spectrophotometer. Glucose and glycerol concentrations were measured using enzymatic kits.

\section{Plasmid construction for chromosome integration}

The primers used to construct $Y$. lipolytica expression vectors are listed in Table 1 . The orotidine $5^{\prime}$-phosphate decarboxylase (URA3) containing the LoxP site was obtained by PCR amplification with primers URA3-1 and 
Table 1 List of primers used in the study

\begin{tabular}{|c|c|c|}
\hline Primer & Gene/fragment information & Sequence in $5^{\prime}$ to $3^{\prime}$ orientation \\
\hline URA3_1 & Selection marker & $\begin{array}{l}\text { TCC ATA TGA ATT ATG CAT GCA TAA CTT CGT ATA ATG TAT GCT ATA } \\
\text { CGA AGT TAT ACC AAA ATG CCC TCC TAC GAA GCT CGA GC }\end{array}$ \\
\hline URA3_2 & Selection marker & $\begin{array}{l}\text { CCA CAT GTG GGA ATT CAT AAC TTC GTA TAG CAT ACA TTA TAC } \\
\text { GAA GTT ATC GAG AAA CAC AAC AAC ATG CCC CAT TGG AC }\end{array}$ \\
\hline TEF_1 & TEF promoter-XPR2 terminator cassette & $\begin{array}{l}\text { GGA ATT CCG GGTTTA AAC AGA GAC CGG GTT GGC GGC GTA TTT } \\
\text { G }\end{array}$ \\
\hline TEF_XPR2_1 & TEF promoter-XPR2 terminator cassette & $\begin{array}{l}\text { TAG GGT ACC TCT AGA CGT CCA CCC GGG AAG GAT CCTTTG AAT } \\
\text { GAT TCT TAT ACT CAG AAG }\end{array}$ \\
\hline TEF_XPR2_2 & TEF promoter-XPR2 terminator cassette & $\begin{array}{l}\text { CTT CTG AGT ATA AGA ATC ATT CAA AGG ATC CTT CCC GGG TGG } \\
\text { ACG TCT AGA GGT ACC CTA }\end{array}$ \\
\hline XPR2_1 & TEF promoter-XPR2 terminator cassette & $\begin{array}{l}\text { CCA CAT GTG GAC GTC GAC GCC ACC TAC AAG CCA GAT TTT CTA } \\
\text { TTT AC }\end{array}$ \\
\hline ARS_1 & Yarrowia automatously replicating sequence & TCC ATA TGC CAG TCT ACA CTG ATT AATTTT CGG G \\
\hline ARS_2 & Yarrowia automatously replicating sequence & TTG CAT GCA TAA GCT AAA AGT AAC TCG CAG CGC A \\
\hline rDNA_1 & Ribosomal DNA integration site & $\begin{array}{l}\text { TCC ATA TGG CGG CCG CGG GTC CGG CTG CCA GTT GCC CAG CCG } \\
\text { CCA G }\end{array}$ \\
\hline rDNA_2 & Ribosomal DNA integration site & ATG CAT GCT GGT GGT AGT AGC AAA TAT TCA AAT G \\
\hline rDNA_3 & Ribosomal DNA integration site & $\begin{array}{l}\text { GCG TCG ACG TTG GCG CGC CTG CTT CGG TAT GAT AGG AAG AGC } \\
\text { CG }\end{array}$ \\
\hline rDNA_4 & Ribosomal DNA integration site & CCA CAT GTG CGG CCG CGG CAG ACA CTG CGT CGC TCC GTC CAC \\
\hline TEF_Casset_1 & TEF promoter & GCG TCG ACA GAG ACC GGG TTG GCG GCG TATTTG \\
\hline TEF_Casset_2 & TEF promoter & TTG GCG CGC CAG AGA CCG GGT TGG CGG CGT ATT TG \\
\hline XPR2-Casset_1 & XPR2 terminator & TTG GCG CGC CGC CAC CTA CAA GCC AGA TTT TCT ATTTAC \\
\hline ACAT_F: & Acetyl-CoA thiolase & GGA TCC ATG CGA CTC ACT CTG CCC CGA CTT \\
\hline ACAT_R & Acetyl-CoA thiolase & CCT AGG CTA CTC GAC AGA AGA GAC CTT CTT G \\
\hline NphT7_F & Acetoacetyl CoA synthase & GGA TCC ATG ACT GAT GTC CGA TTC CGC ATT ATC \\
\hline NphT7_R & Acetoacetyl CoA synthase & CCT AGG TTA CCA CTC AAT CAG AGC GAA GCT \\
\hline HMGS_F & HMG-CoA synthase & GGA TCC ATG TCG CAA CCC CAG AAC GTT GG \\
\hline HMGS_R & HMG-CoA synthase & CCT AGG CTA CTG CTT GAT CTC GTA CTT TCG \\
\hline $\mid \mathrm{PI} \_\mathrm{F}$ & IPP isomerase & TGA TCA ATG CTT CGT TCG TTG CTC AGA GGC \\
\hline IPI_R & IPP isomerase & CCT AGG TCA CGC TTC GTT GAT GTG ATG CAC \\
\hline tHMGR_F & Truncated $\mathrm{HMG}-\mathrm{CoA}$ reductase & TGA TCA ATG CGA GAA GTT GTG CGA ACC CAG \\
\hline tHMGR-R & Truncated HMG-CoA reductase & CCT AGG CTA TGA CCG TAT GCA AAT ATT CGA AC \\
\hline MK_F & Mevalonate kinase & TGA TCA ATG GAC TAC ATC ATT TCG GCG C \\
\hline MK_R & Mevalonate kinase & CCT AGG CTA ATG GGT CCA GGG ACC GAT \\
\hline PMK_F & Phosphomevalonate kinase & GGA TCC ATG ACC ACC TAT TCG GCT CCG GG \\
\hline PMK_R & Phosphomevalonate kinase & CCT AGG CTA CTT GAA CCC CTT CTC GAG CC \\
\hline MPD_F & Mevalonate pyrophosphate decarboxylase & TGA TCA ATG ATC CAC CAG GCC TCC ACC ACC \\
\hline MPD_R & Mevalonate pyrophosphate decarboxylase & CCT AGG CTA CTT GCT GTT CTT CAG AGA ACC \\
\hline FPPS_F & $\begin{array}{l}\text { Fusion gene of farnesyl pyrophosphate synthase and geranyl } \\
\text { pyrophosphate synthase }\end{array}$ & GGA TCC ATG CGG GAT CCA TGT CCA AGG CGA \\
\hline FPPS::GGPPS_R & $\begin{array}{l}\text { Fusion gene of farnesyl pyrophosphate synthase and geranyl } \\
\text { pyrophosphate synthase }\end{array}$ & $\begin{array}{l}\text { AAA TCC GCG CTG TTA TAA TCC ATA GAA CCA CCA CCC TTC TGT } \\
\text { CGC TTG TAA ATC TTG G }\end{array}$ \\
\hline FPPS::GGPPS_F & $\begin{array}{l}\text { Fusion gene of farnesyl pyrophosphate synthase and geranyl } \\
\text { pyrophosphate synthase }\end{array}$ & $\begin{array}{l}\text { CCA AGA TTT ACA AGC GAC AGA AGG GTG GTG GTT CTA TGG ATT } \\
\text { ATA ACA GCG CGG ATTT }\end{array}$ \\
\hline GGPPS_R & $\begin{array}{l}\text { Fusion gene of farnesyl pyrophosphate synthase and geranyl } \\
\text { pyrophosphate synthase }\end{array}$ & C CTA GGT CAC TGC GCA TCC TCA AAG TAC TTT C \\
\hline OfCCD1_F & Carotenoid cleavage dioxygenase 1 & GGA TCC ATG GGT ATG CAG GGC GAG GAT GCT \\
\hline OfCCD1_R & Carotenoid cleavage dioxygenase 1 & CCT AGG TTA CAC CTT AGC CTG CTC CTG GAG C \\
\hline
\end{tabular}


URA3-2 using Y. lipolytica genomic DNA as template. The resulting URA3 fragment was cloned into the pUC57 vector to generate the YAL-URA3 construct. A TEF promoter-XPR2 terminator cassette was constructed by stitching two nucleic acid fragments by PCR amplification. First, a 406 bp nucleic acid fragment comprising the TEF promoter was amplified with the primers TEF-1 and TEF_XPR2-1. A 134 bp nucleic acid fragment comprising the XPR2 terminator was amplified with the primers TEF_XPR2-2 and XPR2-1. The TEF-XPR2 cassette was obtained with primers TEF-2 and XPR2-2. The cassette was then cloned into the YAL-URA3 vector to generate the YAL-URA3-TEF-XPR2 vector. A 572 bp nucleic acid fragment comprising the recombination site rDNA1 and a 822 bp nucleic acid fragment comprising the recombination site rDNA2 were amplified using primers rDNA_1 and rDNA_2, and rDNA_3 and rDNA_4, respectively, using $Y$. lipolytica genomic DNA as a template. The nucleic acid fragments comprising rDNA1 and rDNA2 were cloned into the YAL-URA3-TEF-XPR2 construct to yield YAL-rDNA-URA3-TEF-XPR2.

The pathway of $\beta$-carotene biosynthesis was reconstructed in $Y$. lipolytica by expressing two enzymes: phytoene dehydrogenase (carB) and bifunctional lycopene cyclase/phytoene synthase (carRP) of M. circinelloides. An additional gene encoding the carotenoid cleavage dioxygenase (CCD1) of $O$. fragrans is required for $\beta$-ionone synthesis. These three genes were synthesized based on $Y$. lipolytica-preferred codon usage and cloned into YAL-rDNA-URA3-TEF-XPR2 to form the plasmids YAL-rDNA-URA3-TEF-carB, YAL-rDNAURA3-TEF-carRP, and YAL-rDNA-URA3-TEF-OfCCD1, respectively. The TEF-carRP-XPR2 and TEF-OfCCD1XPR2 cassettes were obtained by PCR amplification with primers TEF_Casset_1 and XPR2-Casset_1 and TEF_Casset_2 and XPR2-Casset_1, respectively. The amplified carRP expression cassette was cloned into YAL-rDNA-URA3-TEF-carB to generate YAL-rDNAURA3-TEF-carB-carRP for $\beta$-carotene synthesis and YAL-rDNA-URA3-TEF-carB-carRP-OfCCD1 for $\beta$-ionone synthesis. In order to enhance precursor supply, eight native genes were over-expressed and two heterologous genes were expressed. Each plasmid contained two gene expression cassettes [(1) Acetyl-CoA thiolase $(A C A T)$ and Acetoacetyl CoA synthase (NphT7) from Streptomyces; (2) HMG-CoA synthase (HMGS) and Truncated HMG-CoA reductase (tHMGR); (3) Mevalonate kinase $(M K)$ and Phosphomevalonate kinase (PMK); (4) Mevalonate pyrophosphate decarboxylase $(M P D)$ and IPP isomerase (IPI) from Haemotoccus pluvialis); (5) and a fusion gene of farnesyl pyrophosphate synthase (FPPS) and geranyl pyrophosphate synthase
(GGPPS)]. The plasmids targeted the rDNA sites for integration into the chromosome, which has previously been shown to result in multiple gene copies [48, 49]. Native genes were obtained via PCR amplification using Y. lipolytica genomic DNA as template. Exogenous genes were codon-optimized. As such, all overexpressed genes had the same promoter and terminator (the TEF promoter and XPR2 terminator) [29, 33-35]. The Cre-LoxP system was employed to remove the selection markers after each round of genome integration. The URA3 fragment of YAL-URA3-TEF-XPR2 vector was replaced by the $L E U 2$ fragment to yield the YAL-LEU2-TEF-XPR2. The Y. lipolytica autonomously replicating sequence (ARS) was amplified using primers ARS_1 and ARS_2, and cloned into the YAL-LEU2-TEF-XPR2 vector, to generate vector YAL-LEU2-TEF-XPR2-ARS. The Cre recombinase gene $(\mathrm{Cre})$ was synthesized, and cloned into the YAL-LEU2TEF-XPR2-ARS vector, to yield the YAL-LEU2-Cre vector for marker excision.

\section{Transformation and marker excision}

For $\beta$-carotene synthesis, the Y. lipolytica strain CLIB138 was transformed with linear plasmid YAL-rDNA-URA3TEF-carB-carRP by NotI on SD-Uracil selection plates according to the Frozen EZ Yeast Transformation Kit II (Zymo Research, CA). The positive colonies were identified via color screening and HPLC analysis. The strain was subsequently transformed with YAL-LEU-Cre for URA3 marker excision as describe in Fickers et al. [50]. The resulting strain without the marker selection gene was used for the next round of transformations. To enhance GGPP supply, ten genes were transformed into the $\beta$-carotene producing strain via five rounds of transformations and marker rescue. The Ura+ transformants were identified by PCR with each gene specific primer and TEF promoter primer. The resulting engineered strain was transformed with the linearized plasmid YALrDNA-URA3-TEF-carB-carRP-ofCCD1 for $\beta$-ionone synthesis, and to incorporate additional copies of $\operatorname{car} B$ and $\operatorname{carRP}$ to possibly increase $\beta$-carotene accumulation.

\section{Tests for nutrient effects on the $\beta$-carotene production}

Shaking tube cultures $(5 \mathrm{~mL})$ were used to screen nutrient effects. YNB medium was supplemented with combinations of $2 \mathrm{~g} / \mathrm{L}$ of a secondary carbon source (acetate, ethanol, glucose, glycerol, pyruvate) or $0.5 \mathrm{~g} / \mathrm{L}$ of an amino acid (isoleucine, leucine, methionine, phenylalanine, valine) added at either 0,18 or $24 \mathrm{~h}$ (see Additional file 1 ). The resulting $\beta$-carotene titers and yields per biomass were normalized to the control (control conditions: cells grown in YNB medium supplemented with an additional $2 \mathrm{~g} / \mathrm{L}$ glucose) and analyzed by a machine 
learning model. Based on model predictions, the defined medium $\mathrm{YNB}(+)$ was formulated by augmenting $\mathrm{YNB}$ medium with $2 \mathrm{~g} / \mathrm{L}$ glycerol, $0.25 \mathrm{~g} / \mathrm{L}$ valine and $0.25 \mathrm{~g} / \mathrm{L}$ isoleucine (total of $0.5 \mathrm{~g} / \mathrm{L}$ amino acid), and with $0.5 \mathrm{~g} / \mathrm{L}$ ethanol added during the middle-log phase. An Eppendorf BioFlo 120 Bioprocess Control Station (2 L working volume) was used for $\beta$-carotene fermentation ( $\mathrm{pH} 5.5$ controlled by $30 \% \mathrm{NaOH}$; dissolved oxygen minimum set at $20 \%$, with $100 \%$ as the initial DO, $0 \%$ baseline noise). The $\beta$-carotene YPD medium fermentation was fed-batch $(250 \mathrm{~mL} 10 \times$ YPD medium followed by a total of $\sim 225 \mathrm{~g}$ glucose, batch added in equal aliquots at hours 72,96 , and 120).

\section{Machine learning of shaking tube data}

Machine learning was performed in the Anaconda JupyterLab Python environment with the Scikit-learn packaging [51]. Data was preprocessed to form two categories: a high performance group (top $10 \% \beta$-carotene production) and a 'background' group. A Gradient Boosting Classifier with 25 trees and five features was trained on $70 \%$ of the data. The remaining $30 \%$ of data was used to predict the accuracy of the model. All the accuracy metrics and feature importance scores reported were based on permutation method to avoid sampling biases. To better evaluate the effect of each feature on predicting, we implemented a procedure which is similar to the one described in Azure Machine Learning Studio to evaluate feature importance. The fitting procedure was described as below:

Step 1: We randomly split the data into a training set and a test set (70\% for training).

Step 2: Trained a Gradient Boosting Classifier with 25 trees and five features on the training data set (the accuracy of this model on the test data set is reported).

Step 3: For feature importance, we randomly shuffled the first feature while keeping the other features unchanged, recalculated the model prediction (built in Step 2) on the shuffled test data, and recorded the difference in prediction accuracy between the original test set and shuffled test set. The difference is recorded as the importance score for the first feature in this round. We sequentially implemented this procedure for all features and obtained their importance scores.

Step 4: Repeated Step 1 to Step 3 for 1000 times.

Step 5: Averaged the accuracy and feature importance scores recorded in each round.

The python code for these will be available upon request.

\section{$\beta$-Ionone fermentations}

For $\beta$-ionone fermentation, $25 \mathrm{~mL}$ YPD medium seed cultures were used to inoculate an Eppendorf BioFlo 120 Bioprocess Control Station (2 L working volume) containing $850 \mathrm{~mL}$ of YPD medium (initial OD 0.7). $150 \mathrm{~mL}$ of dodecane was used as the initial organic overlay layer and was added in a batch manner to maintain an approximately $10 \%$ dodecane layer. During 6 day fermentation period, $250 \mathrm{~mL} 10 \times$ YPD was gradually added to promoted biomass growth during day 1 followed by feeding $500 \mathrm{~g} / \mathrm{L}$ glucose at rate of $0.2 \mathrm{~mL} / \mathrm{min}\left(28-30^{\circ} \mathrm{C}, \mathrm{pH} 5.50\right.$, DO 20\%). During fermentation, time-course samples were taken to monitor cell growth and ionone production. In other tests, the fed-batch fermentations were run by continuously feeding cell with $50 \%$ glycerol (instead of glucose) with the addition of extra valine $(0.25 \mathrm{~g} / \mathrm{L})$ and isoleucine $(0.25 \mathrm{~g} / \mathrm{L})$ in the medium. Finally, we tested strain production stability. Specifically, the engineered $\beta$-carotene and $\beta$-ionone strains were plated on YPD agar from their respective glycerol stocks. Then a single colony was re-plated. The colonies from second set of plates were used to inoculate $25 \mathrm{~mL}$ YPD medium in shaking flasks. After 24-26 h of cultivation, the strains were routinely sub-cultured (starting $\mathrm{OD}_{600} \sim 0.02$ ) until the loss of production was observed.

\section{$\beta$-Carotene and $\beta$-ionone measurements}

$\beta$-Carotene was measured following a reported method [36]. Briefly, centrifuged biomass pellets were washed and re-suspended in $1 \mathrm{~mL}$ of dodecane. 250-400 $\mu \mathrm{g}$ of acid washed glass beads were added and the mixture was vortexed until no orange pellet was observed. $200 \mu \mathrm{L}$ of dodecane was then transferred to a Corning Inc. 96-well assay plate (black plate, clear bottom with lid). The absorbance was measured on a Tecan Infinite M200 Pro Microplate Reader at $454 \mathrm{~nm}$. A standard curve was used to quantify $\beta$-carotene. To confirm $\beta$-carotene production, an Alliance 2996 HPLC (Waters, MA) equipped with a 2476 photodiode array detector was employed. Samples were separated by reverse-phase chromatography on a YMC carotenoid column (particle size $5 \mu \mathrm{m} ; 250 \times 4.6 \mathrm{~mm}$ ) isocratically using a mobile phase of methyl-t-butyl ether/methanol/ethyl acetate (in a 40:50:10 vol/ $\mathrm{vol} / \mathrm{vol}$ ratio) at a flow rate of $0.5 \mathrm{~mL} / \mathrm{min}$ for $25 \mathrm{~min}$.

$\beta$-Ionone in dodecane layers were directly measured by GC-MS. $\beta$-ionone in the aqueous medium and cell pellets were extracted using dodecane before GC-MS analysis. An Agilent Technologies gas chromatography (7820A GC system equipped with a HP- 5 column) and a single quadruple mass analyzer (5977E MSD) were used for ionone analyses. Operational conditions were as follows: the $\mathrm{GC}$ was initially held at $100{ }^{\circ} \mathrm{C}$ for $3 \mathrm{~min}$, and then 
ramped at a rate of $3{ }^{\circ} \mathrm{C} / \mathrm{min}$ until $145{ }^{\circ} \mathrm{C}$ was reached. The inlet port was held at $250{ }^{\circ} \mathrm{C}$. The MS source was held at $230{ }^{\circ} \mathrm{C}$ while the quadruple was at $150{ }^{\circ} \mathrm{C}$. Helium gas was used as a carrier gas. $\beta$-Ionone retention times were confirmed using both standards and published mass spectrums [52]. Standard curves were built to quantify the extracted ionone concentration.

\section{Isotopic labeling and analysis}

The seed cultures inoculated a $5 \mathrm{~mL}$ YNB medium containing $10 \mathrm{~g} / \mathrm{L} \mathrm{U}_{-}{ }^{13} \mathrm{C}$ (fully) labeled glucose at a $0.5 \mathrm{vol} /$ vol \%. In the early exponential phase, cultures were pulsed with either unlabeled glycerol or ethanol to final concentrations of $5 \mathrm{~g} / \mathrm{L}$ or isoleucine or valine to final concentrations of $0.5 \mathrm{~g} / \mathrm{L}$. Cells were harvested $24 \mathrm{~h}$ after the pulse. Free metabolites were analyzed by LC-MS in Joint Bio-Energy Institute. Specifically, cell metabolism was quickly quenched using liquid $\mathrm{N}_{2}$ and centrifuged at $5500 \mathrm{rpm}$ for $5 \mathrm{~min}$ at $0{ }^{\circ} \mathrm{C}$ [53]. LC-MS was operated as previously described [53]. The only differences were that the mobile phase was changed to $20 \mathrm{mM}$ ammonium carbonate (Sigma-Aldrich, St. Louis, MO, USA) in water (solvent A) and $20 \mathrm{mM}$ ammonium carbonate in $70 \%$ acetonitrile and $30 \%$ water (solvent B); the column compartment was set to $40{ }^{\circ} \mathrm{C}$, and the liquid chromatography gradient was changed as follows: Linearly decreased from $100 \%$ B to $70 \%$ B in 9 min, decreased from $70 \%$ B to $60 \% \mathrm{~B}$ in $2.8 \mathrm{~min}$, increased from $60 \% \mathrm{~B}$ to $100 \% \mathrm{~B}$ in $0.2 \mathrm{~min}$, and held at $100 \% \mathrm{~B}$ for a further $10 \mathrm{~min}$. The total LC run time was $22 \mathrm{~min}$. A flow rate of $0.2 \mathrm{~mL} / \mathrm{min}$ was used throughout.

\section{Additional file}

Additional file 1. Small tube medium test data. This file contains the small tube medium test data labels for Fig. 2 as well as the data input for machine learning analysis.

\begin{abstract}
Abbreviations
CCD: carotenoid cleavage dioxygenase; DCW: dry cell weight; FPPS: farnesyl pyrophosphate synthase; GGPP: geranylgeranyl pyrophosphate; GGPPS: geranyl pyrophosphate synthase; GPS: geranyl phosphate synthase; GRAS: generally recognized as safe; HMGS: 3-hydroxy-3-methyl-glutaryl-coenzyme A synthase; IPI: isopentenyl/dimethylallyl diphosphate isomerase; IPP: isopentenyl pyrophosphate; MK: Mevalonate kinase; MPD: Mevalonate pyrophosphate decarboxylase; PMK: Phosphomevalonate kinase; rpm: rotations per minute; tHMGR: Truncated HMG-CoA reductase; Y. lipolytica: Yarrowia lipolytica; YNB: yeast nitrogen base; YPD: yeast extract-peptone-dextrose.
\end{abstract}

\section{Authors' contributions}

YW initiated the project. JC, YW, and YT designed fermentation experiments. $J C, Y W$, and JN performed strain optimization, fermentations and product recovery. JC, VB, and EB performed LC-MS analysis. JC, QC, and YT performed machine learning/statistics. All authors wrote and approved the final version of the paper. Arch Innotek, LLC provides the engineered strain and owns the complete IP. All authors read and approved the final manuscript.

\begin{abstract}
Author details
${ }^{1}$ Department of Energy, Environmental and Chemical Engineering, Washington University, St. Louis, MO 63130, USA. ${ }^{2}$ Lawrence Berkeley National Laboratory, Berkeley, CA 94720, USA. ${ }^{3}$ Department of Mathematics, Statistics and Computer Science, University of Illinois at Chicago, Chicago, IL 60607, USA. ${ }^{4}$ Present Address: Monsanto Company, St Louis, MO 63167, USA. ${ }^{5}$ Arch Innotek, LLC, 4320 Forest Park Ave, St. Louis, MO 63108, USA.
\end{abstract}

\section{Acknowledgements}

We wish to thank Mary Abernathy for helping with metabolite analysis.

\section{Competing interests}

The authors declare that they have no competing interests.

\section{Availability of data and materials}

All data generated or analyzed during this study are included in this published article.

\section{Consent for publication}

All authors agreed to publish this article.

\section{Ethics approval and consent to participate} Not applicable.

\section{Funding}

This work is supported by two National Science Foundation grants (IIP 1722313 and MCB 1616619).

\section{Publisher's Note}

Springer Nature remains neutral with regard to jurisdictional claims in published maps and institutional affiliations.

Received: 18 May 2018 Accepted: 24 August 2018

Published online: 01 September 2018

\section{References}

1. Cheng AX, Lou YG, Mao YB, Lu S, Wang LJ, Chen XY. Plant terpenoids: biosynthesis and ecological functions. J Integr Plant Biol. 2007;49:179-86.

2. Gershenzon J, Dudareva N. The function of terpene natural products in the natural world. Nat Chem Biol. 2007:3:408-14.

3. Goto T, Takahashi N, Hirai S, Kawada T. Various terpenoids derived from herbal and dietary plants function as PPAR modulators and regulate carbohydrate and lipid metabolism. PPAR Res. 2010;2010:483958.

4. Caputi L, Aprea E. Use of terpenoids as natural flavouring compounds in food industry. Recent Patents Food Nutr Agric. 2011;3:9-16.

5. Wang G, Tang W, Bidigare RR. Terpenoids as therapeutic drugs and pharmaceutical agents. In: Zhang L, Demain AL, editors. Natural products: drug discovery and therapeutic medicine. Totowa: Humana Press; 2005. p. $197-227$

6. Shobha RI, Rajeshwari CU, Andallu B. Chapter 9-Oxidative stress and antioxidant herbs and spices in cancer prevention A2-Preedy, Victor. In: Cancer. San Diego: Academic Press; 2014:91-100.

7. Beekwilder J, van der Meer IM, Simic A, Uitdewilligen J, van Arkel J, de Vos RC, Jonker H, Verstappen FW, Bouwmeester HJ, Sibbesen O, et al. Metabolism of carotenoids and apocarotenoids during ripening of raspberry fruit. BioFactors. 2008;34:57-66.

8. Lalko J, Lapczynski A, McGinty D, Bhatia S, Letizia CS, Api AM. Fragrance material review on beta-ionone. Food Chem Toxicol. 2007;45(Suppl 1):S241-7.

9. Duncan RE, Lau D, El-Sohemy A, Archer MC. Geraniol and beta-ionone inhibit proliferation, cell cycle progression, and cyclin-dependent kinase 2 activity in MCF-7 breast cancer cells independent of effects on HMGCoA reductase activity. Biochem Pharmacol. 2004;68:1739-47.

10. Liu JR, Sun XR, Dong HW, Sun CH, Sun WG, Chen BQ, Song YQ, Yang BF. beta-lonone suppresses mammary carcinogenesis, proliferative activity and induces apoptosis in the mammary gland of the Spraque-Dawley rat. Int J Cancer. 2008;122:2689-98. 
11. Nacke C, Huttmann S, Etschmann MM, Schrader J. Enzymatic production and in situ separation of natural beta-ionone from beta-carotene. J Ind Microbiol Biotechnol. 2012;39:1771-8.

12. Berger RG. Biotechnology of flavours - the next generation. Biotechnol Lett. 2009;31:1651.

13. Tenenbaum DJ. Food vs. fuel: diversion of crops could cause more hunger. Environ Health Perspect. 2008;116:A254-7.

14. Cataldo VF, López J, Cárcamo M, Agosin E. Chemical vs. biotechnological synthesis of C13-apocarotenoids: current methods, applications and perspectives. Appl Microbiol Biotechnol. 2016;100:5703-18.

15. Schempp FM, Drummond L, Buchhaupt M, Schrader J. Microbial cell factories for the production of terpenoid flavor and fragrance compounds. J Agric Food Chem. 2018;66:2247-58.

16. Zhang C, Chen X, Lindley ND, Too HP. A "plug-n-play" modular metabolic system for the production of apocarotenoids. Biotechnol Bioeng. 2018;115:174-83.

17. Beekwilder J, van Rossum HM, Koopman F, Sonntag F, Buchhaupt M, Schrader J, Hall RD, Bosch D, Pronk JT, van Maris AJ, Daran JM. Polycistronic expression of a beta-carotene biosynthetic pathway in Saccharomyces cerevisiae coupled to beta-ionone production. J Biotechnol. 2014;192:383-92.

18. Lopez J, Essus K, Kim IK, Pereira R, Herzog J, Siewers V, Nielsen J, Agosin E. Production of beta-ionone by combined expression of carotenogenic and plant CCD1 genes in Saccharomyces cerevisiae. Microb Cell Fact. 2015;14:84.

19. Barth $\mathrm{G}$, Gaillardin C. Physiology and genetics of the dimorphic fungus Yarrowia lipolytica. FEMS Microbiol Rev. 1997;19:219-37.

20. Tai M, Stephanopoulos G. Engineering the push and pull of lipid biosynthesis in oleaginous yeast Yarrowia lipolytica for biofuel production. Metab Eng. 2013;15:1-9.

21. Zhu Q, Jackson EN. Metabolic engineering of Yarrowia lipolytica for industrial applications. Curr Opin Biotechnol. 2015;36:65-72.

22. Löbs A-K, Schwartz C, Wheeldon I. Genome and metabolic engineering in non-conventional yeasts: current advances and applications. Synthetic Syst Biotechnol. 2017:2:198-207.

23. Gao S, Tong Y, Zhu L, Ge M, Zhang Y, Chen D, Jiang Y, Yang S. Iterative integration of multiple-copy pathway genes in Yarrowia lipolytica for heterologous beta-carotene production. Metab Eng. 2017;41:192-201.

24. Gao S, Tong Y, Zhu L, Ge M, Jiang Y, Chen D, Yang S. Production of beta-carotene by expressing a heterologous multifunctional carotene synthase in Yarrowia lipolytica. Biotechnol Lett. 2017;39:921-7.

25. Larroude M, Celinska E, Back A, Thomas S, Nicaud JM, Ledesma-Amaro R. A synthetic biology approach to transform Yarrowia lipolytica into a competitive biotechnological producer of beta-carotene. Biotechnol Bioeng. 2018;115:464-72

26. Yaguchi A, Spagnuolo M, Blenner M. Engineering yeast for utilization of alternative feedstocks. Curr Opin Biotechnol. 2018;53:122-9.

27. Czech A, Smolczyk A, Ognik K, Kiesz M. Nutritional value of Yarrowia lipolytica yeast and its effect on growth performance indicators in piglets. Ann Anim Sci. 2016;16:1091

28. Czajka J, Wang Q, Wang Y, Tang YJ. Synthetic biology for manufacturing chemicals: constraints drive the use of non-conventional microbial platforms. Appl Microbiol Biotechnol. 2017;101:7427-34.

29. Cao X, Lv Y-B, Chen J, Imanaka T, Wei L-J, Hua Q. Metabolic engineering of oleaginous yeast Yarrowia lipolytica for limonene overproduction. Biotechnol Biofuels. 2016;9:214.

30. Okamura E, Tomita T, Sawa R, Nishiyama M, Kuzuyama T. Unprecedented acetoacetyl-coenzyme A synthesizing enzyme of the thiolase superfamily involved in the mevalonate pathway. Proc Natl Acad Sci. 2010;107:11265

31. Reiling KK, Yoshikuni Y, Martin VJ, Newman J, Bohlmann J, Keasling JD. Mono and diterpene production in Escherichia coli. Biotechnol Bioeng 2004;87:200-12.

32. Martin VJJ, Pitera DJ, Withers ST, Newman JD, Keasling JD. Engineering a mevalonate pathway in Escherichia coli for production of terpenoids. Nat Biotechnol. 2003:21:796.
33. Rico J, Pardo E, Orejas M. Enhanced production of a plant monoterpene by overexpression of the 3-hydroxy-3-methylglutaryl coenzyme A reductase catalytic domain in Saccharomyces cerevisiae. Appl Environ Microbiol. 2010;76:6449-54.

34. Broker JN, Muller B, van Deenen N, Prufer D, Schulze Gronover C. Upregulating the mevalonate pathway and repressing sterol synthesis in Saccharomyces cerevisiae enhances the production of triterpenes. Appl Microbiol Biotechnol. 2018;102:6923-34.

35. Guo X, Sun J, Li D, Lu W. Heterologous biosynthesis of (+)-nootkatone in unconventional yeast Yarrowia lipolytica. Biochem Eng J. 2018;137:125-31.

36. Reyes LH, Gomez JM, Kao KC. Improving carotenoids production in yeast via adaptive laboratory evolution. Metab Eng. 2014;21:26-33.

37. Friedman JH. Greedy function approximation: a gradient boosting machine. Ann Stat. 2001;29:1189-232.

38. Hastie T, Tibshriani R, Friedman J. The elements of statistical learning. 2nd ed. New York: Springer; 2009.

39. Fichan I, Larroche C, Gros JB. Water solubility, vapor pressure, and activity coefficients of terpenes and terpenoids. J Chem Eng Data. 1999;44:56-62.

40. O'Neil MJ, editor. The Merck index: an encyclopedia of chemicals, drugs, and biologicals. Cambridge: Royal Society of Chemistry; 2013. p. 940.

41. Davies FK, Work VH, Beliaev AS, Posewitz MC. Engineering limonene and bisabolene production in wild type and a glycogen-deficient mutant of Synechococcus sp. PCC 7002. Front Bioeng Biotechnol. 2014;2:21.

42. Yaegashi J, Kirby J, Ito M, Sun J, Dutta T, Mirsiaghi M, Sundstrom ER, Rodriguez A, Baidoo E, Tanjore D, et al. Rhodosporidium toruloides: a new platform organism for conversion of lignocellulose into terpene biofuels and bioproducts. Biotechnol Biofuels. 2017;10:241.

43. Koch B, Schmidt C, Daum G. Storage lipids of yeasts: a survey of nonpolar lipid metabolism in Saccharomyces cerevisiae, Pichia pastoris, and Yarrowia lipolytica. FEMS Microbiol Rev. 2014:38:892-915.

44. Wu G, Yan Q, Jones JA, Tang YJ, Fong SS, Koffas MA. Metabolic burden: cornerstones in synthetic biology and metabolic engineering applications. Trends Biotechnol. 2016;34:652-64.

45. Juminaga D, Baidoo EE, Redding-Johanson AM, Batth TS, Burd H, Mukhopadhyay A, Petzold CJ, Keasling JD. Modular engineering of L-tyrosine production in Escherichia coli. Appl Environ Microbiol. 2012;78:89-98.

46. Jiang W, Qiao JB, Bentley GJ, Liu D, Zhang F. Modular pathway engineering for the microbial production of branched-chain fatty alcohols. Biotechnol Biofuels. 2017;10:244.

47. Chen X, Zhu P, Liu L. Modular optimization of multi-gene pathways for fumarate production. Metab Eng. 2016:33:76-85.

48. Le Dall MT, Nicaud JM, Gaillardin C. Multiple-copy integration in the yeast Yarrowia lipolytica. Curr Genet. 1994;26:38-44.

49. Juretzek T, Le Dall M, Mauersberger S, Gaillardin C, Barth G, Nicaud J. Vectors for gene expression and amplification in the yeast Yarrowia lipolytica. Yeast. 2001:18:97-113.

50. Fickers P, Le Dall MT, Gaillardin C, Thonart P, Nicaud JM. New disruption cassettes for rapid gene disruption and marker rescue in the yeast Yarrowia lipolytica. J Microbiol Methods. 2003;55:727-37.

51. Pedregosa F, Varoquaux G, Gramfort A, Michel V, Thirion B, Grisel $O$, Blondel M, Prettenhofer P, Weiss R, Dubourg V, et al. Scikit-learn: machine learning in Python. J Mach Learn Res. 2011;12:2825-30.

52. Walwil AM. The Mass Spectra Analysis for a-ionone and $\beta$-ionone. Int J Chem. 2017:9:61

53. Hollinshead WD, Rodriguez S, Martin HG, Wang G, Baidoo EE, Sale KL, Keasling JD, Mukhopadhyay A, Tang YJ. Examining Escherichia coli glycolytic pathways, catabolite repression, and metabolite channeling using Deltapfk mutants. Biotechnol Biofuels. 2016;9:212.

54. Haddad SA, Lindegren CC. A method for determining the weight of an individual yeast cell. Appl Microbiol. 1953;1:153-6. 